\title{
Visual inspection with Acetic Acid (VIA) as an Alternative to Colposcopy for detection of Cervical Carcinoma in low recourses setting
}

\author{
Masand D', Dubey $\mathbf{S}^{2}$ \\ ${ }^{1}$ Dr Deepa Masand, Associate Professor, Department of Obstetrics \& Gynaecology, ${ }^{2}$ Dr Smarika Dubey, Post graduate \\ student, Obstetric \& Gynaecology. Both are affiliated with NIMS Medical College, Shobhanagar, Jaipur, Rajasthan, India
}

\begin{abstract}
Objective: To evaluate visual inspection with acetic acid ( VIA ) as an alternative to colposcopy in detection of cervical carcinoma in low resource setting. Introduction: Cervical cancer is a major public health problem in developing countries, with India itself accounting for one-fifth of the global burden of the disease. In low resource settings, screening of CIN and carcinoma cervix by colposcopy can be replaced by cheaper and easily available visual methods like VIA, which has the high sensitivity to detect any grade of dysplasia. Method: VIA was performed in two hundred symptomatic women as a pre colposcopy attending the gynaecology department at National Institute of Medical Science ( NIMS ) Hospital Jaipur. Sensitivity of VIA was calculated. Result: Out of 200 patients VIA was positive in $48(24 \%)$ cases. In VIA positive cases, Colposcopy was abnormal in 30 ( 62.5\%). In VIA negative cases colposcopy was abnormal in only in 2 (1.3\%) cases. 32 cases with abnormal colposcopic finding were subjected to biopsy. Conclusion: The high Sensitivity of $86.6 \%$ of VIA shows that most of the preinvasive and invasive lesion were picked up by VIA. Thus VIA could be valuable in detection of precancerous lesion of the cervix in low resource setting.
\end{abstract}

Key Words: Visual Inspection with Acetic Acid, Colposcopy, Cervical Cancer, Low Resource Setting

\section{Introduction}

Cervical cancer is an avoidable cause of death among women with 528000 new cases every year. Cervical cancer is the fourth most common cancer affecting women worldwide, after breast, colorectal and lung cancers ; it is most notable in the lower-resource countries of sub-Saharan Africa. It is also the fourth most common cause of cancer death (266000 deaths in 2012) in women worldwide. Almost $70 \%$ of the global burden falls in areas with lower levels of development and more than one fifth of all new cases are diagnosed in India [1].

Cervical cancer is preceded by a long phase of cytological changes, known as cervical intraepithelial neoplasia ( CIN ) and takes a long period of 15-20 years before the invasive cancer develops [2] .

Thus cervical cancer can be prevented if cellular changes are detected and treated in early stage. In western countries, cervical cancer incidence and death have been brought under control using organised cytology based screening program (pap smear)[3]. Though cytology (pap smear) is reliable, the laboratory infrastructure and

Manuscript received: $24^{\text {th }}$ June 2014

Reviewed: $07^{\text {th }}$ July 2014

Author Corrected: $14^{\text {th }}$ July 2014

Accepted for Publication: $26^{\text {th }}$ July 2014 logistic including technical expertise may not be available in low resource setting. The cost factor in testing for human papillomavirus using DNA testing, coupled with requirement of trained manpower makes this option non-viable for developing countries [4]. India with its highest share of global burden of cervical cancer has to implement a population based cervical cancer control programme to reduce the number of death [5]. Visual inspection using acetic acid (VIA) has emerged as a promising, cost effective, non-cytology based, "see and treat" alternative for economically underprivileged geographic regions. The present study was undertaken to find the value of VIA in picking up pre-invasive or invasive cancer in an abnormal cervix. All VIA positive cases were further evaluated with a colposcopy. A directed biopsy was taken in cases with abnormal colposcopy

\section{Material and Methods}

This prospective study was conducted in department of Obstetrics and Gynaecology National Institute of Medical Science (NIMS) Jaipur (Rajasthan). 200 symptomatic women over a period of one year attending Gynaecology clinic were studied. Approval of the institutional ethical committee and informed consent was 
obtained from each women. Relevant obstetric and gynaecological history was obtained and recorded.

Inclusion criteria:

Age - $>25$ yrs

Marrital life $>3$ yrs

Parous

Non Pregnant

Symptomatic cases

\section{Exclusion criteria:}

Virgin

Pregnant

In menstrual phase

H/o hysterectomy

Confirmed cases of carcinoma cervix

After General physical examination, local examination, cervix was exposed by Cusco's vaginal speculum. Any evidence of infection, ectopy, any visible growth etc were examined. Then 3-5\% acetic acid solution was applied to the cervix for 1 minute (with the help of swab stick ) and then the cervix was carefully inspected for any acetowhite lesions, particularly in the transformation zone. The normal squamous epithelium of the cervix is pink. On application of acetic acid, cervical intraepithelial neoplasia (CIN) lesion takes on white colour due to increased nuclear proteins and cytokeratins in the cervical epithelium [7].

\section{Criteria for classifying via positive}

- Location of lesion

- Colour intensity of acetowhite lesion

- Borders and demarcation of acetowhite lesion

- $\quad$ Size of lesion

- Extension of acetowhite lesion

After VIA patient were then examined by Colposcope with $12 \mathrm{X}-15 \mathrm{X}$ magnification. Biopsy was taken only from those who showed abnormal colposcopic appearances. Colposcopy and biopsy was taken as a reference standard for statistical calculation.

\section{Observation}

Results are tabulated and discussed as follow.

Table 1: Demographic distribution of cases

\begin{tabular}{|c|c|c|c|c|c|}
\hline \multirow[t]{2}{*}{ Age Group (years) } & \multirow[t]{2}{*}{ No. of Women } & \multicolumn{4}{|c|}{ Parity } \\
\hline & & $1-2$ & $3-4$ & $5-6$ & 7 or above \\
\hline $18-24$ & 12 & 07 & 01 & 04 & 00 \\
\hline $29-34$ & 62 & 01 & 37 & 23 & 01 \\
\hline $35-44$ & 94 & 02 & 64 & 28 & 00 \\
\hline $45-54$ & 25 & 00 & 12 & 11 & 02 \\
\hline$>54$ & 07 & 00 & 03 & 01 & 03 \\
\hline
\end{tabular}

Out of 200 symptomatic women, most women belong to age group of 35-44 yrs and parity between 3- 4 . The mean age of cases was 38.175 yrs with mean parity of 4.19 . The commonest presenting complaint was vaginal discharge $(62 \%$ of the patient). Other presenting complaints were intermenstrual bleeding ( $25 \%$ of the patient), postcoital bleeding ( $8 \%$ of the patient) and postmenopausal bleeding ( $5 \%$ of the patient).

On visual inspection of cervix with 3-5\% acetic acid, 48 cases (24\%) were VIA positive, Of these 30 cases $(62.5 \%)$ had abnormal colposcopic finding and 18 cases (37.5\%) had normal colposcopic finding. 152 cases (76\%) were VIA negative, Of these 2 cases (1.3\%) had abnormal colposcopic finding and 150 cases (98.6 5) had normal colposcopic finding .

Table 2: Correlations of histopathology with VIA result

\begin{tabular}{|l|l|l|l|}
\hline \multirow{2}{*}{ HPR } & VIA & Positive & \multirow{2}{*}{ Total } \\
\cline { 2 - 4 } & Negative & 10 & 12 \\
\hline CIN 1 & 2 & 4 & 4 \\
\hline CIN 2 & & 2 & 2 \\
\hline CIN 3 & & 1 & 1 \\
\hline Invasive Cancer & & 13 & 13 \\
\hline Normal/Negative & & 30 & 32 \\
\hline Total & 2 & & \\
\hline
\end{tabular}


30 cases of VIA positive correlated with positive histopathological finding. Remaining 18 VIA positive cases have normal histopathology.

Table 3: Correlations of colposcopy and histopathology

\begin{tabular}{|l|l|l|l|}
\hline & Colposcopy & & \\
\hline HPR & Negative & Positive & Total \\
\hline CIN 1 & & 12 & 12 \\
\hline CIN 2 & & 4 & 4 \\
\hline CIN 3 & & 2 & 2 \\
\hline Invasive Cancer & & 1 & 1 \\
\hline Chronic cervicitis & & 13 & 13 \\
\hline Total & 0 & 32 & 32 \\
\hline
\end{tabular}

As per table all the cases with abnormal colposcopy findings have positive histopathology results.

Table 4: Correlations of colposcopy and VIA with biopsy result

\begin{tabular}{|l|l|l|l|l|}
\hline \multirow{2}{*}{ HPR } & \multicolumn{2}{|l|}{ Sensitivity } & PPV \\
\cline { 2 - 3 } & VIA & Colposcopy & VIA & \multirow{2}{*}{ Colposcopy } \\
\hline CIN 1 & $10 / 12$ & $12 / 12$ & \\
\hline CIN 2 & $4 / 4$ & $4 / 4$ & & \\
\cline { 1 - 3 } CIN 3 & $2 / 2$ & $2 / 2$ & & \\
\hline Invasive Cancer & $1 / 1$ & $1 / 1$ & $17 / 30$ & $19 / 32$ \\
\hline Overall & $17 / 19$ & $19 / 19$ & & \\
\hline
\end{tabular}

It is clear from table that for CIN 1 stage sensitivity of VIA is less. For advanced lesion sensitivity of VIA \& colposcopy was $100 \%$.

Table 5: Sensitivity and positive predictive value of VIA and colposcopy

\begin{tabular}{|l|l|l|l|l|}
\hline & \multicolumn{2}{|l|}{ Sensitivity } & \multirow{2}{*}{ PPV } \\
\cline { 1 - 3 } HPR & VIA & Colposcopy & \multirow{2}{*}{ VIA } & \\
\cline { 1 - 2 } CIN 1 & $83.33 \%$ & $100.00 \%$ & & \\
\cline { 1 - 2 } CIN 2 & $100.00 \%$ & $100.00 \%$ & & \\
\cline { 1 - 3 } CIN 3 & $100.00 \%$ & $100.00 \%$ & & \\
\cline { 1 - 3 } Invasive Cancer & $100.00 \%$ & $100.00 \%$ & $56.67 \%$ & $5.375 \%$ \\
\hline Overall & $89.47 \%$ & $100.00 \%$ & \\
\hline
\end{tabular}

The most sensitive test for all grades of CIN and invasive cancer is colposcopy followed by VIA which is less sensitive in CIN1.

Pearson Chi-Square $=101.609 \quad \mathrm{DF}=1 \quad$ 'p' value $<0.001$

Kappa value $=0.691 \quad$ 'p' value $<0.001$

As above table shows that there was $15 \%$ of positive cases and $75 \%$ of negative cases were showing agreement. On application of Chi-square test and kappa statistics there were significant association (Chi-square -101.609 and $\mathrm{p}$ value $<$ $0.001)$ and statistically significant $(\mathrm{k}-0.691, \mathrm{p}<0.001)$. 
Thus from above table it is clear that there is statistically significant association between colposcopy and VIA. Thus VIA is an alternative of Colposcopy.

\section{Discussion}

Cervical cancer is a potentially preventable cancer. It is preceded by premalignant lesions which may take 5-15 years to progress to invasive cancer. If detected and treated timely, pre-invasive disease has nearly 100 per cent cure rate with simple surgical procedure, while advanced cancers have less than 35 per cent survival rates [8].

However, in developing countries like India, universal screening has not been achieved. The main screening method (Pap smear) is available to a small percentage of population. Cytology based screening programmes are difficult to organize owing to limited infrastructure, trained personnel and funds [9].

It has been estimated that in India, even with a major effort to expand cytology services, it will not be possible to screen even one-fourth of the population once in a lifetime [10].

Moreover, screening programmes in India are mostly institution based and are restricted to urban centres [11]. Thus, in developing countries, there is a need for alternative strategies for early detection of premalignant cervical lesions.

Thus VIA is a cost effective method which can differentiate a normal cervix from a precancerous cervix with reasonable accuracy. Hence, if practiced by gynaecologists routinely, it would help to avoid many panic hysterectomies. Besides, in the absence of organized cytology screening program and limited availability of colposcopy facilities, VIA can be propagated on a wider scale.

In the present study, women from all the age group were included. The mean age was 38.17 years and majority were parous women. This observation agrees with the studies of singh kavita $\mathrm{N}$ et al [12].

$\mathrm{N}$ Yusuf et al studies correspond well with this study that CIN is more prone in sexually active women. Therefore, Screening for cervical carcinoma should start ideally at the onset of sexually activity and all the women should be screened at least once by the age of 30-35 years.

In our study sensitivity of VIA was $89.47 \%$ which shows that most of the pre-invasive and invasive lesions were picked up by VIA. Only two patients were missed (false negative), one with post -menopausal bleeding where transformation zone receded inside and other case was of adenocarcinoma.

Shankarnarayanan and Mahe [13] have published results from a randomized intervention trial in India comparing VIA to cytology and to HPV DNA testing, and found that all three had similar detection rates of CIN 2 and 3 lesions, and the range of sensitivity for VIA was $67-79 \%$ and specificity $49-86 \%$.

The higher sensitivity of our study can be explained on the basis that the study was done on a group of women in susceptible age group (mean $38.48 \pm 8.9$ years). Secondly, the VIA was performed using colposcope halogen bulb (24V 250 Watt) by a gynaecologist and not by a nurse or a paramedical person.

In JHPIEGO cervical cancer project at Zimbabwe, VIA was done by trained nurses and paramedical person on 10,934 women and was found to have sensitivity of $76.4 \%$ and specificity of $64 \%$ in picking up pre-invasive lesions [14].

The study also inferred that higher test qualities of VIA are likely to be observed under better service delivery conditions like good lighting, examination tables and specula, and more standardized VIA training. They observed that in more than $75 \%$ of cases when a lesion was found on colposcopy or biopsy, it was also visible on VIA [15].

Ottaviano and Torre [16], in the first study of its kind, have published results of simultaneous VIA and colposcopy on 2,400 unselected patients with normal or abnormal cervical cytology.

In our study, VIA and biopsy correlation is less for CIN1 which is $83.3 \%$., but the sensitivity increased up to $100 \%$ in picking up CIN 2, CIN 3 which is indeed a true cancer precursor and early invasive cancer.Colposcopic magnification is a complimentary method to VIA and not essential to identify a cervix with higher grade lesions.

VIA can also guide a practicing gynaecologist regarding the site to be biopsied. Although colposcope accurately identifies the most abnormal area for biopsy,VIA will demarcate the site precisely and give better results than a 
blind biopsy of ectocervix, in absence of facilities for colposcopy [17].

In our study it is clear that there is statistically significant association between colposcopy and VIA. Thus VIA is an alternative of Colposcopy.

\section{Conclusion}

VIA is attractive test in low resource settings like India. It is simple, inexpensive low technology test that requires minimal infrastructure.

The majority of women belonging to low socioeconomic status, remain devoid of any screening test, thus time has come to integrate VIA based screening program at the primary care level of health services and to down stage cancer cervix in our country.

\section{Funding: Nil}

\section{Conflict of interest: Nil}

\section{Permission from IRB: Yes}

\section{References}

1. Ferlay J, Soerjomataram I, Ervik M, DikshitR,Eser S, Mathers C, Rebelo M, Parkin DM, Forman D, Bray, F (2013). GLOBOCAN 2012 v1.0, Cancer Incidence and Mortality Worldwide: IARC Cancer Base No. 11 [Internet]. Lyon, France:International Agency for Research on Cancer. Available from: http://globocan.iarc.fr, accessed on 26/August/2014.

2. Shafi MI, Welton K. Colposcopy and cervical intraepithelial neoplasia. Obstet Ggynaecol Reprod Med 2007; 17(6): 173-180.

3. American Cancer Society. Cancer facts and figures. Atlanta, Ga: American Cancer Society; 2011. Available from: www.cancer.org/research/2011-cancer-facts-andfigures.pdf. accessed on 26/August/2014.

4. Nelson R. HPV Testing most successful method for cervical cancer screening in developing countries. N Engl J Med 2009;360:1385-94.

5. Basu P, Chowdhury D. Cervical cancer screening and HPV Vaccination : a comprehensive approach to cervical cancer control. Indian J Med Res, 2009 sep;130:241-246.

6. Sarian LO, Derchian SF, Naud P et al. Evaluation of visual inspection with acetic acid, Lugol's iodine, cervical cytology and HPV testing as cervical screening tools in latin America . J Med Screen. 2005;12(3):142-9

7. Carr KC, Sellem MW. Cervical cancer screening in low resources settings using visual inspection with acetic acid. J Midwifery Womens Health. 2004 JulAug;49(4):329-37

8. Dinshaw KA, Shastri SS. Screening for cervical cancer in India. Natl Med J India. 2001 Jan-Feb;14(1):1-3.

9. Shastri SS, Dinshaw K, Amin G, Goswami S, Patil S, Chinoy R, et al. Concurrent evaluation of visual, cytological and HPV testing as screening methods for early detection of cervical neoplasia in Mumbai, India. Bull World Health Organ. 2005 Mar;83(3):186-94.

10. Juneja A, Sehgal A, Sharma S, Pandey A. Cervical cancer screening in India: Strategies revisited. Indian J Med Sci. 2007 Jan;61(1):34-47.

11. Misra JS, Gupta HP, Das V. Assessing the feasibility of single lifetime PAP smear evaluation between 41-50 years of age as strategy for cervical cancer control in developing countries from our 32 years of experience of hospital-based routine cytological screening. Diagn Cytopathol. 2004 Dec;31(6):376-9.

12. Singh Kavita N, More Shefali. Visual inspection of cervix with acetic acid in early diagnosis of cervical intraepithelial neoplasia ( CIN) and early cancer cervix. j obstet Gynecol India.2010;60(1) January /

February:55-60

13. Shankarnarayanan R, Mahe C. Cervical cancer control indeveloping world. Indian J Gynecol Oncol 2004;4:5-13.

14. Visual inspection with acetic acid for cervical cancer screening: test qualities in primary - care setting. University of Zimbabwe /JHPIEGO Cervical Cancer Project. Lancet 1999;353:869-73.

15. Ottaviano M, La Torre P. Examination of the cervix with the naked eye using acetic acid test. Am J Obstet Gynecol. 1982 May 15;143(2):139-42.

16. Hopman EH, Kenemans P, Helmerhorst TJ. Positive predictive rate of colposcopic examination of the cervix uteri: an overview of literature. Obstet Gynecol Surv. 1998 Feb;53(2):97-106. 
17. Bharani Bharti 1, Phatak Satish R Acetic acid visualization of the cervix an alternative to colposcopy in evaluation of cervix at risk $\mathrm{J}$ Obstet Gynecol India 2005;55(6):530-533.

\section{How to cite this article?}

Masand D, Dubey S. Visual inspection with Acetic Acid (VIA) as an Alternative to Colposcopy for detection of Cervical Carcinoma in low recourses setting. Int $J$ Med Res Rev 2014;2(5):444- 449. https://doi:10.17511/ijmrr.2014.i05.08 